\title{
2-D AFFINE GENERALIZED FRACTIONAL FOURIER TRANSFORM
}

\author{
Jian-Jiun Ding Soo-Chang Pei \\ Department of Electrical Engineering, National Taiwan University, Taipei, Taiwan, R.O.C \\ Email address: pei@cc.ee.ntu.edu.tw.
}

\begin{abstract}
The 2-D Fourier transform has been generalized into the 2-D separable fractional Fourier transform (replaces 1-D Fourier transform by 1-D fractional Fourier transform for each variable) and the 2-D separable canonical transform (further replaces the fractional Fourier transform by canonical transform) in [3]. It also has been generalized into the 2-D unseparable fractional Fourier transform with 4 parameters in [6]. In this paper, we will introduce the 2-D affine generalized fractional Fourier transform (AGFFT). It has even further generalized these 2-D transforms. We will show it can deal with many problems that can't be dealt by these 2-D transforms and extend their utility.
\end{abstract}

\section{INTRODUCTION}

The canonical transform [1] is defined as:

$$
\mathrm{O}_{\mathrm{F}}^{(a, b, c, d)}(f(t))=\int_{-\infty}^{\infty} K_{(a, b, c, d)}(u, t) \cdot f(t) \cdot d t
$$

where

$$
K_{(a, b, c, d)}(u, t)=\sqrt{\frac{1}{2 \pi|b|}} e^{\frac{j}{2} \frac{d}{b} u^{2}} e^{-j \frac{u}{b} t} e^{\frac{j}{2} \frac{a}{b} t^{2}}
$$

and ad-bc $=1$. It is the generalization of Fourier transform, and is a useful tool for optical system analysis. It has the additivity property as:

$$
\begin{aligned}
& O_{F}^{(e, f, g, h)}\left(O_{F}^{(a, b, c, d)}(f(t))\right)=O_{F}^{(n, o, p, q)}(f(t)) \\
& \text { where }\left[\begin{array}{ll}
n & o \\
p & q
\end{array}\right]=\left[\begin{array}{ll}
a & b \\
c & d
\end{array}\right] \cdot\left[\begin{array}{ll}
e & f \\
g & h
\end{array}\right]
\end{aligned}
$$

The additivity property here ignores the constant phase multiplication. In 1980, Namias introduced the fractional Fourier transform (FRFT)[2]. It is the special case of canonical transform that $\{a, b, c, d\}=\{\cos \alpha, \sin \alpha,-\sin \alpha, \cos \alpha\}$. It is very useful, and has been used for many applications such as filter design, pattern recognition, and optical system analysis.

Recently, in [3], they introduce the 2-D fractional Fourier transform and canonical transform. The 2-D canonical transform they introduce is equivalent to the following equation:

$$
\begin{aligned}
& \mathrm{O}_{\mathrm{F}}^{(\mathrm{a}, \mathrm{b}, \mathrm{c}, \mathrm{d}, \mathrm{e}, \mathrm{f}, \mathrm{g}, \mathrm{h})}(\mathrm{g}(\mathrm{x}, \mathrm{y})) \\
= & \int_{-\infty}^{\infty} \int_{-\infty}^{\infty} \mathrm{K}_{(\mathrm{a}, \mathrm{b}, \mathrm{c}, \mathrm{d})}(\mathrm{f}, \mathrm{x}) \mathrm{K}_{(\mathrm{e}, \mathrm{f}, \mathrm{g}, \mathrm{h})}(\mathrm{h}, \mathrm{y}) \mathrm{g}(\mathrm{x}, \mathrm{y}) \mathrm{dxdy}
\end{aligned}
$$

where $K_{(a, b, c, d)}(f, x), K_{(e, f, g, h)}(h, y)$ are all defined as equ. (2). It can be viewed as the combination of 2 1-D canonical transforms.
In this paper, we call it as the 2-D separable canonical transform. The 2-D fractional Fourier transform they define is the special case that $\{a, b, c, d\}=\{\cos \alpha, \sin \alpha,-\sin \alpha, \cos \alpha\}$ and $\{e, f, g, h\}$ $=\{\cos \beta, \sin \beta,-\sin \beta, \cos \beta\}$. Then, in [6], they introduce the 2-D unseparable fractional Fourier transform. It is the 2-D fractional Fourier transform of $f\left[\left(\cos \theta_{1} x+\sin \theta_{1} y\right) / \cos \left(\theta_{1}-\theta_{2}\right), \quad-\sin \theta_{2} x+\right.$ $\left.\cos \theta_{2} y / \cos \left(\theta_{1}-\theta_{2}\right)\right]$, and has the free dimension of 4 .

In this paper, we will further generalize these 2-D transforms into the 2-D affine generalized fractional Fourier transform (AGFFT). The 2-D AGFFT we introduce is unseparable, and has the free dimension of 10 . It has extended the utility of 2-D transforms described above. In this paper we will all ignore the constant phase multiplication.

\section{DEFINITION OF THE 2-D AGFFT}

The 2-D affine generalized fractional Fourier transform (AGFFT) is defined as [4]:

$$
\begin{aligned}
& O_{F}^{(A, B, C, D)}(g(x, y)) \\
&=\int_{-\infty}^{\infty} \int_{-\infty}^{\infty} K_{(A, B, C, D)}(f, h, x, y) \cdot g(x, y) \cdot d x d y \\
& \text { where } \quad A=\left[\begin{array}{ll}
a_{11} & a_{12} \\
a_{21} & a_{22}
\end{array}\right] \quad B=\left[\begin{array}{ll}
b_{11} & b_{12} \\
b_{21} & b_{22}
\end{array}\right] \\
& C=\left[\begin{array}{ll}
c_{11} & c_{12} \\
c_{21} & c_{22}
\end{array}\right] \quad D=\left[\begin{array}{ll}
d_{11} & d_{12} \\
d_{21} & d_{22}
\end{array}\right]
\end{aligned}
$$

represents the 16 parameters of 2D AGFFT (Here we restrict all the parameters to be real), and the kernel is:

$$
\begin{aligned}
& K_{(A, B, C, D)}(f, h, x, y) \\
& =\frac{e^{\frac{j}{2 \operatorname{det}(B)}\left(k 1 \cdot f^{2}+k 2 \cdot f \cdot h+k 3 \cdot h^{2}\right)} \quad \frac{j}{2 \pi \sqrt{|\operatorname{det}(B)|}\left(p 1 \cdot x^{2}+p 2 \cdot x \cdot y+p 3 \cdot y^{2}\right)}}{e^{2 \operatorname{det}(B)}} \\
& \text { where } \quad \begin{array}{ll}
k 1=d_{11} b_{22}-d_{12} b_{21} & k 2=2\left(-d_{11} b_{12}+d_{12} b_{11}\right) \\
k 3=-d_{21} b_{12}+d_{22} b_{11} & p 1=a_{11} b_{22}-a_{21} b_{12} \\
p 2=2\left(a_{12} b_{22}-a_{22} b_{12}\right) & p 3=-a_{12} b_{21}+a_{22} b_{11}
\end{array}
\end{aligned}
$$

We note, the 2-D separable canonical transform is the special case that $a_{12}=a_{21}=b_{12}=b_{21}=c_{12}=c_{21}=d_{12}=d_{21}=0$. The following constraints must all be satisfied for the 2-D AGFFT.

$$
A^{T} C=C^{T} A \quad B^{T} D=D^{T} B
$$




$$
\mathrm{A}^{\mathrm{T}} \mathrm{D}-\mathrm{C}^{\mathrm{T}} \mathrm{B}=\mathrm{I}
$$

Thus, there are 16 paraneters and 6 constraints (2 for equ. (7) and 4 for equ. (8)), so the free dimension of the 2-D AGFFT is 10. In contrast, the free dimension for 1-D canonical transform is 3 , for the 2-D separable canonical transform is 6 , and for the 2-D unseparable fractional Fourier transform defined in [6] is 4 . The additive property for the 2-D AGFFT is

$$
\begin{aligned}
& \mathrm{O}_{\mathrm{F}}^{\left(\mathrm{A}^{\prime}, \mathrm{B}^{\prime}, \mathrm{C}^{\prime}, \mathrm{D}^{\prime}\right)}\left(\mathrm{O}_{\mathrm{F}}^{(\mathrm{A}, \mathrm{B}, \mathrm{C}, \mathrm{D})}(\mathrm{g}(\mathrm{x}, \mathrm{y}))\right) \\
= & \mathrm{O}_{\mathrm{F}}^{\left(\mathrm{A}^{*}, \mathrm{~B}^{\prime}, \mathrm{C}^{\prime}, \mathrm{D}^{\circ}\right)}(\mathrm{g}(\mathrm{x}, \mathrm{y}))
\end{aligned}
$$

where $\left[\begin{array}{ll}\mathrm{A}^{n} & \mathrm{~B}^{\prime \prime} \\ \mathrm{C}^{\prime \prime} & \mathrm{D}^{n}\end{array}\right]=\left[\begin{array}{ll}\mathrm{A}^{\prime} & \mathrm{B}^{\prime} \\ \mathrm{C}^{\prime} & \mathrm{D}^{\prime}\end{array}\right] \cdot\left[\begin{array}{ll}\mathrm{A} & \mathrm{B} \\ \mathrm{C} & \mathrm{D}\end{array}\right]$

There are 10 independent basic operations for the 2-D AGFFT (correspond to the 10 free dimension of it).

(1) chirp multiplication for the $\mathrm{x}$-axis

$\left(a_{11}=a_{22}=d_{11}=d_{22}=1, c_{11} \neq 0\right.$, others $\left.=0\right)$

(2) chirp convolution for the $\mathrm{x}$-axis

$$
\left(a_{11}=a_{22}=d_{11}=d_{22}=1, b_{11} \neq 0 \text {, others }=0\right)
$$

(3) scaling in the $x$-axis $\left(a_{11}=1 / d_{11}, a_{22}=d_{22}=1\right.$, others $=0$ )

(4) chirp multiplication for the $y$-axis

(5) chirp convolution for the $y$-axis

(6) scaling in the $y$-axis ((4)-(6) are similar with (1)-(3))

(7) multiplication of $\exp (j \tau x y)$

$\left(\mathrm{a}_{11}=\mathrm{a}_{22}=\mathrm{d}_{11}=\mathrm{d}_{22}=1, \mathrm{c}_{12}=\mathrm{c}_{21}=\tau\right.$, others $\left.=0\right)$

(8) convolution of $\exp (j \eta x y / 4) /(4 \pi \eta)^{\wedge} 0.5$

$\left(a_{11}=a_{22}=d_{11}=d_{22}=1, b_{12}=b_{21}=\eta\right.$, others $\left.=0\right)$

(9) shearing in the $x$-axis $(g(x, y) \rightarrow g(x+p y, y))$

$\left(a_{11}=a_{22}=d_{11}=d_{22}=1,-a_{21}=d_{21}=p\right.$, others $\left.=0\right)$

(10) shearing in the $y$-axis $(g(x, y) \rightarrow g(x, q x+y))$

$\left(a_{11}=a_{22}=d_{11}=d_{22}=1,-a_{12}=d_{12}=q\right.$, others $\left.=0\right)$

We can proof all the 2-D AGFFT can be decomposed as the combination of the above 10 basic operations. We find the former 6 basic operations also exist for the separable 2-D canonical transform, but the later 4 basic operations are not (because for these operations the $\mathrm{x}$-axis and $\mathrm{y}$-axis are not independent).

Because the 2-D AGFFT defined as equ. (5) is very complex, so sometimes we would simplify it. In fact, in many cases we can set $D=0$ because it has only a little contributions for many applications of the 2-D AGFFT except for the optical system analysis. For the filter design, we would set $D=0, B=I, C=-I$ (in this case, $\left.a_{12}=a_{21}\right)$, and modulate $g(x, y)$ before doing the transform, then equ. (5) will become:

$$
\begin{aligned}
& O_{F}^{(A, B, C, D, N)}(g(x, y)) \\
= & \int_{-\infty}^{\infty} \int_{-\infty}^{\infty} e^{-i(f x+h y)} \cdot e^{i\left(a_{11} x^{2}+2 a_{12} x y+a_{22} y^{2}\right) / 2} . \\
& e^{i\left(n_{1} x+n_{2} y\right)} \cdot g(x, y) \cdot d x d y
\end{aligned}
$$

Although this special case only have 5 parameters, but we find it will have almost all the utility of the 2-D AGFFT defined as equ. (5) for the filter design. The transform defined as equ. (10) can filter out all the quadratic type noise of easily.

We can define the 2-D affine generalized fractional correlation as

$$
\begin{aligned}
& Z(f, h)=O_{F}^{(A, B, C, D)}(f(x, y)) \cdot O_{F}^{\left(A^{\prime}, B^{\prime}, C^{\prime}, D^{\prime}\right)}(g(x, y)) \\
& z(x, y)=\operatorname{IFT}(Z(f, h))
\end{aligned}
$$

\section{SOME IMPORTANT PROPERTIES}

We list some important properties for the 2-D AGFFT below. Here we denote $\mathrm{O}_{\mathrm{F}}{ }^{(A, B, C, D)}(\mathrm{g}(\mathrm{x}, \mathrm{y}))$ by $\mathrm{G}_{(\mathrm{A}, \mathrm{B}, \mathrm{C}, \mathrm{D})}(\mathrm{f}, \mathrm{h})$

(1) Reversible property

$$
O_{F}^{\left(D^{T},-B^{T},-C^{T}, A^{T}\right)}\left(O_{F}^{(A, B, C, D)}(f(x, y))\right)=f(x, y)
$$

(2) Orthonormal property

$$
\begin{aligned}
& \int_{-\infty}^{\infty} \int_{-\infty}^{\infty} K_{(A, B, C, D)}(f, h, x, y) K_{(A, B, C, D)}^{*}\left(f^{\prime}, h^{\prime}, x, y\right) d x d y \\
& =\delta\left(f-f^{\prime}, h-h^{\prime}\right)
\end{aligned}
$$

(3) Power preservation property

$$
\int_{-\infty}^{\infty} \int_{-\infty}^{\infty}|g(x, y)| d x d y=\int_{-\infty}^{\infty} \int_{-\infty}^{\infty}\left|G_{(A, B, C, D)}(f, h)\right| d f d h
$$

(4) Relation with the 2-D Wigner distribution function (WDF) The 2-D Wigner distribution function (WDF) is:

$$
\begin{gathered}
W(x, y, f, h)=\int_{-\infty}^{\infty} \int_{-\infty}^{\infty} e^{-j \tau \cdot f} e^{-j \eta \cdot h} g\left(x+\frac{\tau}{2}, y+\frac{\eta}{2}\right) \\
g^{*}\left(x-\frac{\tau}{2}, y-\frac{\eta}{2}\right) \cdot d \tau d \eta
\end{gathered}
$$

Then the 2-D WDF of $g(x, y)$, and of $G_{(A, B, C, D)}(f, h)$ has the following relation:

$$
\begin{aligned}
W_{g}(x, y, f, h)= & W_{G_{(A, B, C, D)}}\left(a_{11} x+a_{12} y+b_{11} f+b_{12} h,\right. \\
& a_{21} x+a_{22} y+b_{21} f+b_{22} h, c_{11} x+c_{12} y \\
& \left.+d_{11} f+d_{12} h, c_{21} x+c_{22} y+d_{21} f+d_{22} h\right)
\end{aligned}
$$

(5) Shifting and modulation property

$$
\begin{aligned}
& O_{F}^{(A, B, C, D)}\left(e^{i \cdot n_{1} x} e^{i \cdot n_{2} y} g\left(x-m_{1}, y-m_{2}\right)\right) \\
= & e^{i \cdot s_{1} f} e^{i \cdot s_{2} h} G_{(A, B, C, D)}\left(f-r_{1}, h-r_{2}\right)
\end{aligned}
$$

where

$$
\left[\begin{array}{l}
r_{1} \\
r_{2} \\
s_{1} \\
s_{2}
\end{array}\right]=\left[\begin{array}{ll}
A & B \\
C & D
\end{array}\right] \cdot\left[\begin{array}{c}
m_{1} \\
m_{2} \\
n_{1} \\
n_{2}
\end{array}\right]
$$

(6) Differentiation and multiplication property

$$
\begin{aligned}
& \mathrm{O}_{\mathrm{F}}^{(\mathrm{A}, \mathrm{B}, \mathrm{C}, \mathrm{D})}\left[\mathrm{h}_{1} \frac{\partial \mathrm{g}(\mathrm{x}, \mathrm{y})}{\partial \mathrm{x}}+\mathrm{h}_{2} \frac{\partial \mathrm{g}(\mathrm{x}, \mathrm{y})}{\partial \mathrm{y}}-\mathrm{h}_{3} \mathrm{jx} \cdot \mathrm{g}(\mathrm{x}, \mathrm{y})\right. \\
& \left.\quad-\mathrm{h}_{4} \mathrm{jx} \cdot \mathrm{g}(\mathrm{x}, \mathrm{y})\right] \\
& =\mathrm{k}_{1} \frac{\partial \mathrm{G}_{(\mathrm{A}, \mathrm{B}, \mathrm{C}, \mathrm{D})}(\mathrm{f}, \mathrm{h})}{\partial \mathrm{f}}+\mathrm{k}_{2} \frac{\partial \mathrm{G}_{(\mathrm{A}, \mathrm{B}, \mathrm{C}, \mathrm{D})}(\mathrm{f}, \mathrm{h})}{\partial \mathrm{h}} \\
& -\mathrm{k}_{3} \mathrm{jf} \cdot \mathrm{G}_{(\mathrm{A}, \mathrm{B}, \mathrm{C}, \mathrm{D})}(\mathrm{f}, \mathrm{h})-\mathrm{k}_{4} \mathrm{jh} \cdot \mathrm{G}_{(\mathrm{A}, \mathrm{B}, \mathrm{C}, \mathrm{D})}(\mathrm{f}, \mathrm{h})
\end{aligned}
$$




$$
\left[\begin{array}{l}
k_{1} \\
k_{2} \\
k_{3} \\
k_{4}
\end{array}\right]=\left[\begin{array}{ll}
A & B \\
C & D
\end{array}\right] \cdot\left[\begin{array}{l}
h_{1} \\
h_{2} \\
h_{3} \\
h_{4}
\end{array}\right]
$$

\section{CALCULATION}

Although the 2-D AGFFT. seems to be very complex, but in fact we can implement it in a simple way. If $\operatorname{det}(B) \neq 0$, then the $2-D$ AGFFT can be decomposed as:

$$
\begin{aligned}
{\left[\begin{array}{ll}
\mathrm{A} & \mathrm{B} \\
\mathrm{C} & \mathrm{D}
\end{array}\right] } & =\left[\begin{array}{cc}
\mathrm{I} & 0 \\
\mathrm{DB}^{-1} & \mathrm{I}
\end{array}\right] \cdot\left[\begin{array}{cc}
0 & \mathrm{I} \\
-\mathrm{I} & 0
\end{array}\right] . \\
& {\left[\begin{array}{cc}
\mathrm{B}^{\mathrm{T}^{-1}} & 0 \\
0 & \mathrm{~B}
\end{array}\right] \cdot\left[\begin{array}{cc}
\mathrm{I} & 0 \\
\mathrm{~B}^{-1} \mathrm{~A} & \mathrm{I}
\end{array}\right] }
\end{aligned}
$$

That is, the 2-D AGFFT can be calculated by the 4 steps: (a) multiply the input function by a quadratic phase function (i.e., $\left.\exp \left(\mathrm{px}^{2}+q x y+r y^{2}\right)\right)$, (b) scaling, (c) 2-D Fourier transform (d) multiplication of a quadratic phase function. This will be easier than directly calculating the integration of equ. (5). And for the digital implementation, we can also follow these steps. We can sample the function after the quadratic phase multiplication and scaling (i.e. after the step a and b), then do the 2-D FFT, and then multiply a quadratic phase function with sampling. The complexity of the digital implementation is proportion to $\left(\mathrm{Nlog}_{2} \mathrm{~N}\right)^{2}$.

\section{APPLICATIONS}

There are many applications for the 2-D AGFFT. For example, the filter design, pattern recognition, optical system analysis, 2-D affine Hilbert transform, signal synthesis, and beam shaping are all its applications. We will illustrate some of them latter.

\section{(1) Filter design}

The filter for the 2-D AGFFT acts as the following formula:

$$
\begin{aligned}
\hat{k}(x, y)=O_{F}^{\left(D^{T},-B^{T},-C^{T}, A^{T}\right)} & \left(O_{F}^{(A, B, C, D)}(k(x, y)) .\right. \\
& \left.O_{F}^{(A, B, C, D)}(z(x, y))\right)
\end{aligned}
$$

This is just the 2-D affine generalized convolution of the signal $k(x, y)$ and the filter $z(x, y)$. There are at least 2 ways to design the filter with 2-D AGFFT. The first is the method of the optimal filter. Because the 2-D AGFFT is also an orthonormal transform, so the formula of the optimal filter for 1-D FRFT [5] can also be applied here with a little modification. Suppose the correlation between the input signal $g(x, y)$ and the received signal $s(x, y)$ and the auto-correlation of the received signal $s(x, y)$ have been known, then the optimal filter is:

$$
z_{\text {opt }}(f, h)=\frac{R_{G_{(A, B, C, D)} S_{(A, B, C, D)}}(f, h, f, h)}{R_{S_{(A, B, C, D)} S_{(A, B, C, D)}}(f, h, f, h)}
$$

where $R_{G(A, B, C, D) S(A, B, C, D)}(f, h, f, h)$ can be calculated from $R_{g s}(x, y$, $\sigma, \tau):$

$$
\begin{aligned}
& R_{G_{(A, B, C, D)} S_{(A, B, C, D)}}(f, h, f, h) \\
= & {\left[\int_{-\infty}^{\infty} \int_{-\infty}^{\infty} \int_{-\infty}^{\infty} \int_{-\infty}^{\infty} K_{(A, B, C, D)}(f, h, x, y) K_{(A, B, C, D)}^{*}(f, h, \sigma, \tau)\right.} \\
& \left.R_{g s}(x, y, \sigma, \tau) d x d y d \sigma d \tau\right]
\end{aligned}
$$

and from similar way, $R_{S(A, B, C, D) S(A, B, C, D)}(f, h, f, h)$ can be calculated from $R_{s s}(x, y, \sigma, \tau)$. If we also know the auto-correlation of the input signal, then we can calculate the mean square error for the optimal filter as:

$$
\begin{aligned}
& \text { MSE }=\int_{-\infty}^{\infty} \int_{-\infty}^{\infty}\left[R_{G_{(A, B, C, D)} G_{(A, B, C, D)}}(f, h, f, h)\right. \\
& -2 \operatorname{Re}\left(z_{o p t}^{*}(f, h) R_{S_{(A, B, C, D} G_{(A, B, C, D)}}(f, h, f, h)\right) \\
& +\left|z_{o p t}(f, h)\right|^{2} R_{S_{(A, B, C, D)} S_{(A, B, C, D)}}(f, h, f, h) \cdot d f d h
\end{aligned}
$$

The second is the method of the ideal type filter. That is, $Z_{(A, B, C, D)}(f, h)$ (the 2-D AGFFT of the filter) equals to 1 in the passband, and equals to 0 in the stopband. In the following we will give an example of the filter of ideal type. Here the signal is the fruit with the size of $256^{*} 256$ (we set the location of $(0,0)$ at the center), and the noise is the function as

$$
n(x, y)=\exp \left(i^{*} 0.001\left(2 x^{2}-10 x y+1.5 y^{2}\right)\right)
$$

In fig. 2, we plot the fruit plus the noise. This noise can't be moved by the separable canonical transforms defined as equ. (4) because of the existence of the term exp(inxy). But we can remove it by the 2-D AGFFT defined as equ. (5) with the parameters $B=-C=I, D=0, a_{11}=-0.004, a_{12}=a_{21}=0.01, a_{22}=-0.003$. In figure 3 , we show the result of the 2-D AGFFT of the fig. 2 with the parameters described above. Then, we use the filter as:

$\mathrm{Z}_{(\mathrm{A}, \mathrm{B}, \mathrm{C}, \mathrm{D})}(\mathrm{f}, \mathrm{h})=1-\delta(\mathrm{f}, \mathrm{h})$

And then, we doing the inverse 2-D AGFFT, and obtain the recovered signal as fig. 4 . We find, the noise has been perfectly removed.

\section{(2) Optical system analysis}

The separable canonical transform has been used for the optical system analysis [3]. It can analyze the cylinder lens with the width variation direction of $x$ or $y$-axis. But for the cylinder lens with the width variation direction other than $x$ and $y$-axis, the separable canonical transform will be fail to analyze it. Instead, we can use the 2-D AGFFT defined as equ. (5) to analyze it. For example, for the optical system in the fig. 1, the transform function of the first cylinder lens is

$$
\mathrm{t}_{1}(\mathrm{x}, \mathrm{y})=\mathrm{e}^{-\mathrm{j} \pi(\mathrm{y} \cos \alpha-\mathrm{x} \sin \alpha)^{2} / \lambda \cdot \mathrm{f} 1}
$$

It just corresponds to the 2-D AGFFT with the parameters $\{\mathrm{I}, 0$, $\Phi, 0\}$ where

$$
\Phi=\left[\begin{array}{cc}
\frac{-\mathrm{k} \sin ^{2} \alpha}{\mathrm{f} 1} & \frac{\mathrm{k} \sin \alpha \cos \alpha}{\mathrm{f} 1} \\
\frac{\mathrm{k} \sin \alpha \cos \alpha}{\mathrm{f} 1} & \frac{-\mathrm{k} \cos ^{2} \alpha}{\mathrm{f} 1}
\end{array}\right]
$$

and $k=2 \pi / \lambda$. And the overall system in fig. 1 corresponds to the 2-D AGFFT with the parameters $\left\{A^{\prime}, B^{\prime}, C^{\prime}, D^{\prime}\right\}$ where : 


$$
\begin{aligned}
& {\left[\begin{array}{ll}
\mathrm{A}^{\prime} & \mathrm{B}^{\prime} \\
\mathrm{C}^{\prime} & \mathrm{D}^{\prime}
\end{array}\right]=\left[\begin{array}{cc}
\mathrm{I} & 0 \\
\Omega & \mathrm{I}
\end{array}\right] \cdot\left[\begin{array}{cc}
\mathrm{I} & \Psi \\
0 & \mathrm{I}
\end{array}\right] \cdot\left[\begin{array}{cc}
\mathrm{I} & 0 \\
\Phi & \mathrm{I}
\end{array}\right]} \\
& \text { where } \Omega=\left[\begin{array}{cc}
0 & 0 \\
0 & -\mathrm{k} / \mathrm{f} 2
\end{array}\right], \quad \Psi=\left[\begin{array}{cc}
\mathrm{d} / \mathrm{k} & 0 \\
0 & \mathrm{~d} / \mathrm{k}
\end{array}\right]
\end{aligned}
$$

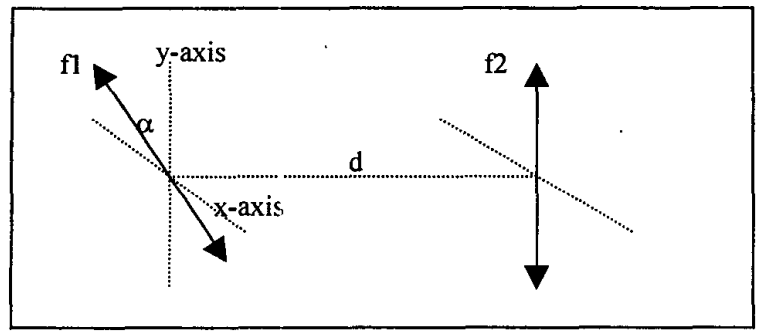

Fig. 1 Optical system with the first cylinder lens has the direction other than $\mathrm{x}$ and $\mathrm{y}$ axis

(3) 2-D affine Hilbert transform

The 2-D affine Hilbert transform acts as the following equation:

$$
\begin{aligned}
\hat{g}(x, y)= & O_{F}^{\left(D^{\mathrm{T}},-B^{\mathrm{T}},-C^{\mathrm{T}}, A^{\mathrm{T}}\right)}\left(H_{\phi, \varphi}(f, h) \cdot\right. \\
& \left.\mathrm{O}_{\mathrm{F}}^{(\mathrm{A}, \mathrm{B}, \mathrm{C}, \mathrm{D})}(\mathrm{g}(\mathrm{x}, \mathrm{y}))\right)
\end{aligned}
$$

where

$$
\mathrm{H}_{\phi, \varphi}(\mathrm{f}, \mathrm{h})=(\cos \phi+\mathrm{j} \sin \phi \cdot \mathrm{u}(\mathrm{f}))(\cos \varphi+\mathrm{j} \sin \varphi \cdot \mathrm{u}(\mathrm{h}))
$$

and $u(f), u(h)$ are step functions. We can choose the parameters as:

$$
\begin{aligned}
{\left[\begin{array}{ll}
\mathrm{A} & \mathrm{B} \\
\mathrm{C} & \mathrm{D}
\end{array}\right] } & =\left[\begin{array}{cc}
\Phi & \mathrm{I} \\
-\mathrm{I} & 0
\end{array}\right] \cdot\left[\begin{array}{cc}
\Omega^{-1} & 0 \\
0 & \Omega
\end{array}\right] \\
\text { where } \Phi & =\left[\begin{array}{cc}
\mathrm{a} 1 & 0 \\
0 & \mathrm{a} 2
\end{array}\right] \quad \Omega=\left[\begin{array}{cc}
1 & \mathrm{v} 1 \\
\mathrm{v} 2 & 1
\end{array}\right]
\end{aligned}
$$

Then, $(1, v 1)$ and $(v 2,1)$ will be the direction of edges to be emphasize by the Hilbert transform. And we can use $\phi$ for $(1, v 1)$ and $\varphi$ for $(\mathrm{v} 2, \mathrm{l})$ to control whether positive or negative edges to be emphasize, and then use al for $(1, \mathrm{vl})$ and $a 2$ for $(\mathrm{v} 2,1)$ to control how much we emphasize the edges.

\section{CONCLUSION}

We have introduced the 2-D AGFFT, some important properties of it, its calculation, and its applications for the filter design, optical system analysis, and Hilbert transform. It generalizes the 2-D separable canonical transform introduced by [3], and mixed it with the space twisting operation. Thus, we believe the 2-D AGFFT can not only extend most of the applications for 1-D fractional Fourier transform, it will also be a useful tool for the pattern recognition and can deal with the space twisting problems.

\section{REFERENCE}

[1] M. Moshinsky and C. Quesne, J. Math. Phys., vol. 12, no. 8, p 1772-1783, Aug, 1971
[2] V. Namias, J. Inst. Maths. Applics., vol. 25, p 241-265, 1980

[3] A. Sahin, H. M. Ozaktas, and D. Mendlovic, Appl. Opt., vol. 37, no. 11, p 2130-2141, 1998

[4] G. B. Folland, "Harmonic Analysis in Phase Space", the Annals of Math. Studies vol. 122, 1989

[5] M. A. Kutay, H. M. Ozaktas, O. Arikan, and L. Onural, IEEE Trans. Signal Processing., vol. 45, no. 5, p 1129-1143, 1997

[6] A. Sahin, M. A. Kutay, and H. M. Ozaktas, Appl. Opt., vol. 37 , no. 23, p 5444-5453, 1998

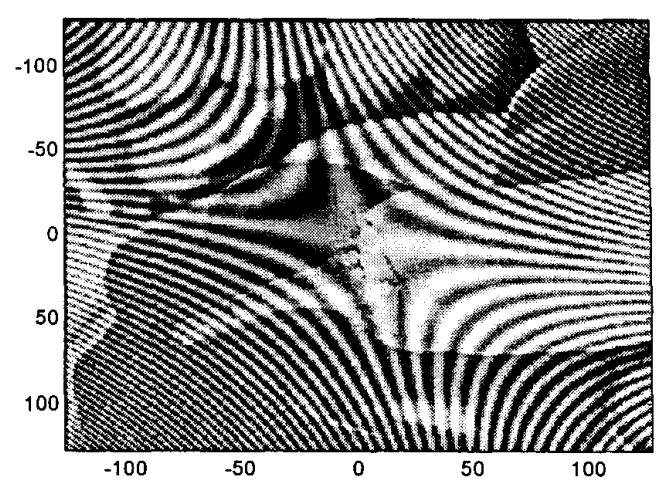

Fig. 2 The signal of fruit plus the noise (defined as equ. (24))

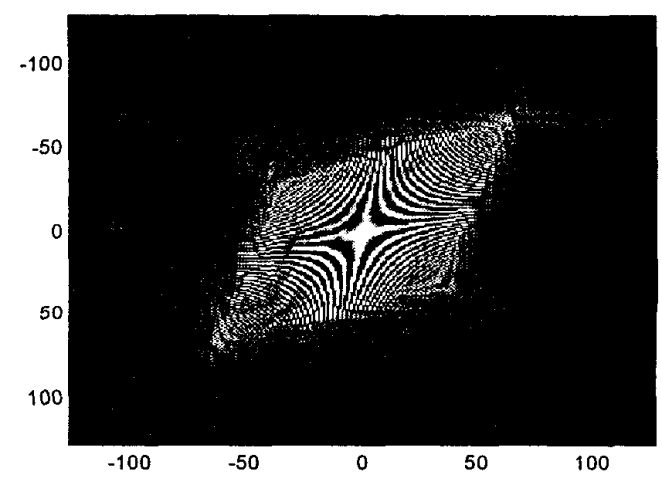

Fig. 3 The 2-D AGFFT of fig. 2

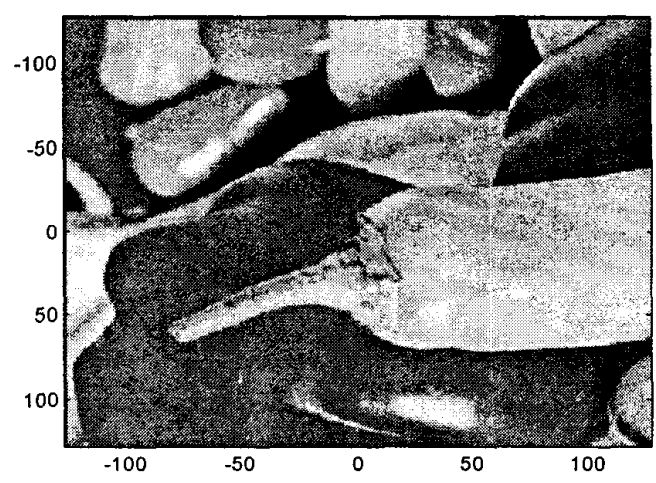

Fig. 4 The recovered signal 